\title{
TWO CASES OF LEIOMYOSARCOMA OF THE LUNG
}

\section{BY}

\author{
J. S. GLENNIE, P. HARVEY, AND P. JEWSBURY \\ From the Thoracic Surgical Unit, Blackpool Victoria Hospital
}

(RECEIVED For PUblication AUgust 25, 1959)

At the beginning of this century, sarcoma of the lung was considered to be a relatively common type of tumour. However, with our gradually accumulating knowledge of histology and the resulting increased understanding of the pathogenesis of tumours, it became apparent that a great proportion of the sarcomas of earlier pathologists were undifferentiated carcinomas. Since the recognition of this fact, the number of pulmonary sarcomas reported in the literature has greatly diminished, and, furthermore, the number of true primary pulmonary leiomyosarcomas recorded has been very small indeed. It is very probable that cases are encountered from time to time in most thoracic surgical units, but these appear to have found scant mention in the literature, and therefore the present cases of pulmonary leiomyosarcoma, one of which was

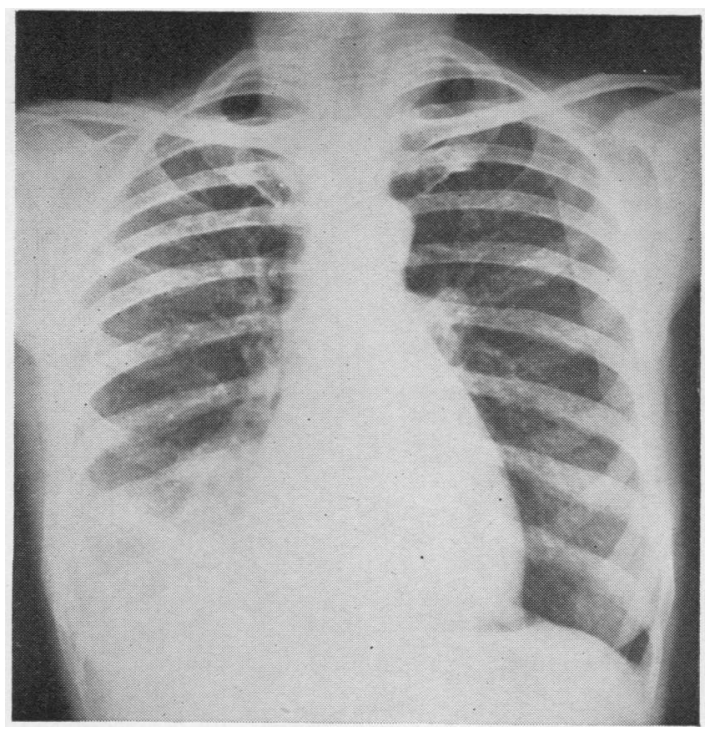

FIG. $1(a)$ and (b).-Antero-posterior and lateral views of Case 1 showing right lower lobe pneumonia with basal effusion in July, 1956. diagnosed by bronchoscopic biopsy and both of which were treated surgically, seem worth recording.

\section{CASE Reports}

CASE 1.-A married woman of 47 was first admitted to an acute medical ward on July 27,1956 , with a pneumonic illness affecting the lower lobe of the right lung. She had been tired and run down for two months, and during the week before admission had suffered increasing cough, with mucoid sputum, dyspnoea, loss of appetite, and slight haemoptysis. Her previous history revealed three episodes of illness requiring treatment in hospital:

(1) In 1951 she had menorrhagia, and an initial suspension operation was followed later by hysterectomy. The uterus when examined by a pathologist showed chronic inflammatory changes in the cervix. There were no fibromyomata in the uterus and no evidence of malignancy.

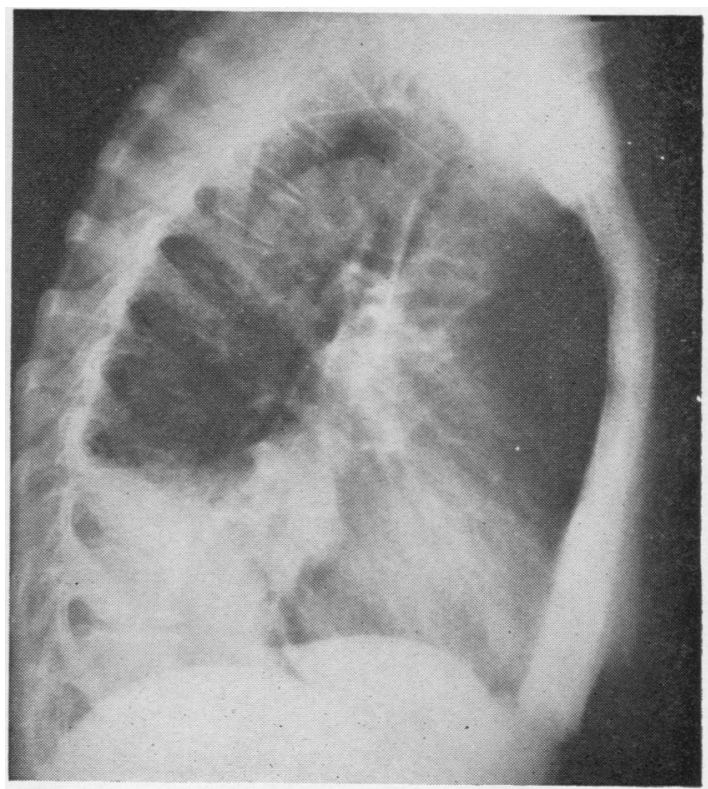

Fig. 1 (b) 


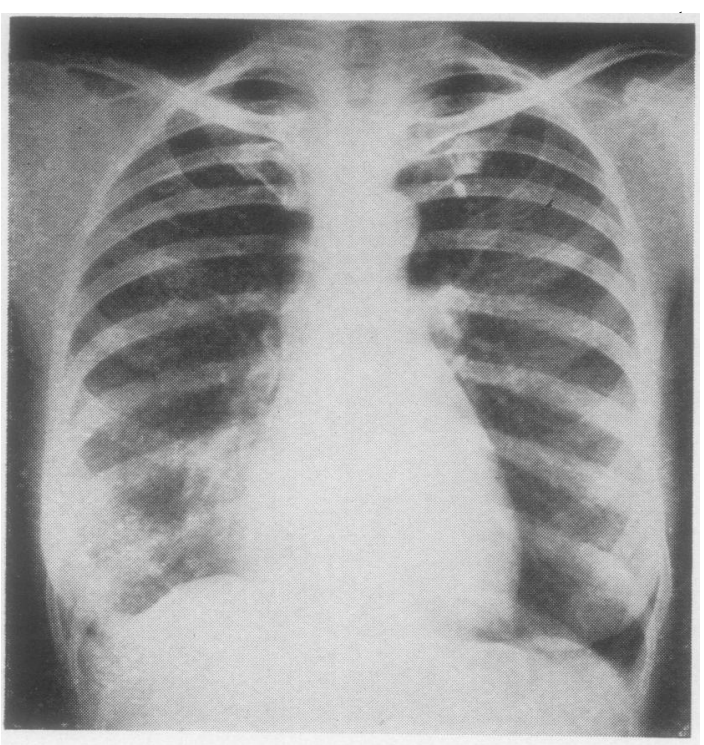

FIG. $2(a)$

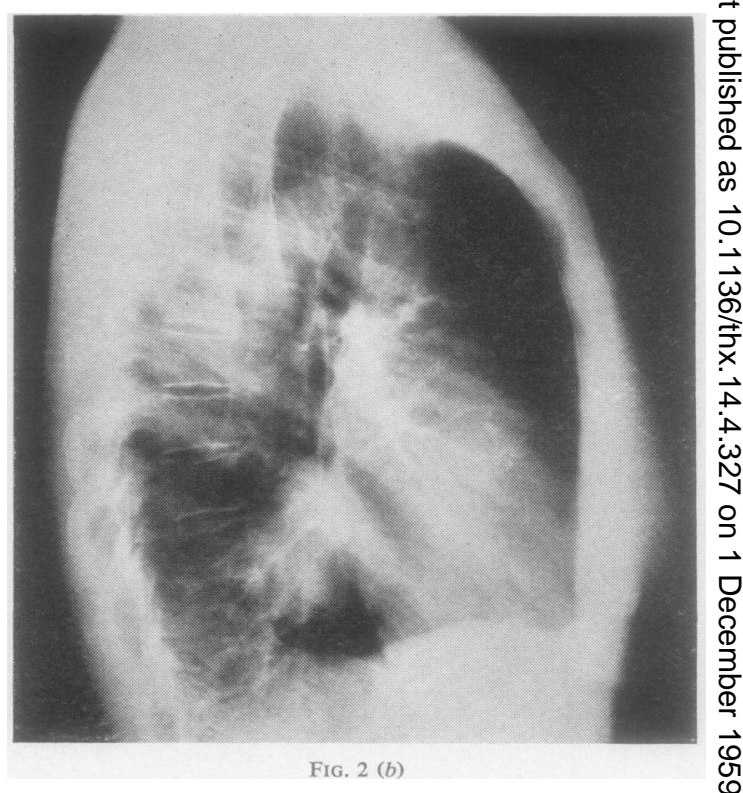

FIG, 2 (b)

FIG. $2(a)$ and $(b)$.-By August, 1956, after chemotherapy, pneumonia has cleared leaving tumour shadow visible at hilum of right lower lobe

(2) In 1952 she had acute hepatitis and recovered after hospital treatment.

(3) In 1953 she had a febrile illness with pains in the neck, shoulders, and lumbar spine and recovered with symptomatic treatment. Liver function tests showed that a slight disturbance of liver function still persisted.

Examination on admission in July, 1956, revealed a thinly built, ill-looking woman with signs of pleurisy and consolidation in the right lower lobe. Radiographs of the chest confirmed a right lower lobar pneumonia with basal effusion (Fig. $1 a$ and $b$ ), which largely cleared with intramuscular penicillin, leaving a hilar shadow interpreted as being due to resolving hilar adenitis.

Re-examination a month later revealed that the cough was still troublesome. Some dullness and diminished air entry persisted at the right base, and new radiographs showed that the right lower lobe basal infiltration had cleared leaving a well-defined lobular shadow in the region of the stem bronchus (Fig. $2 a$ and $b$ ).

Bronchoscopy on October 17 (P.J.) demonstrated obstruction of the right lower lobe stem bronchus immediately below the apical opening, typical of neoplasm. A biopsy was obtained and surprisingly was reported as showing a mesodermal tumour, either fibrosarcoma or leiomyosarcoma.

Right thoracotomy on October 23 (P.J.) revealed a tumour $6 \mathrm{~cm}$. in diameter involving the subapical region of the right lower lobe encroaching on the lung hilum and apparently infiltrating the inferior pulmonary vein as far as the pericardium. The peribronchial glands did not appear to be involved. The pericardium was opened to assess operability, but there was no extension of the tumour palpable within it. It appeared feasible to remove the middle and lower lobes together with a window of pericardium $\mathbb{D}$ around the veins, including the phrenic nerve, and $\underset{\vec{P}}{\vec{P}}$ this was done. Post-operatively suction drainage and $\bar{O}$ pneumoperitoneum elevated the diaphragm sufficiently to seal off an air leak from the lower surface of the upper lobe.

Pathological Report.-The middle and lower right lobes of the right lung and carinal glands were received on October 2, 1956.

The bronchus to the lower lobe was obstructed by $\frac{\dot{\sigma}}{\vec{\sigma}}$ a firm tumour $6.0 \times 6.0 \mathrm{~cm}$. which had penetrated close to the base of the lobe.

Histological examination shows a smooth muscle tumour which is moderately differentiated. There is 0 generally a whorled pattern of the cells, though this is blurred in some fields by local areas of degeneration and necrosis. There are also a few localized areas of round-cell infiltration. There are numerous bizarre N nuclei and mitoses can be seen fairly frequently.

There is no true capsule to the tumour and the ${ }^{N}$ edge is irregular with evidence of invasion round the N circumference. The neoplasm has also invaded a main bronchus right up to the mucosa, though without ulceration. The neoplasm can also be seen eroding bronchial cartilage. In the tumour there are a number of thin-walled vessels.

Dr. A. P. Stout sent the following histological report :

The tumour is a well-differentiated smooth muscle $\stackrel{\Phi}{\overparen{ }}$ tumour. Masson's trichrome stain shows that there $\stackrel{\mathbb{Q}}{\varrho}$ are myofibrils in every cell. Although the tumour is 


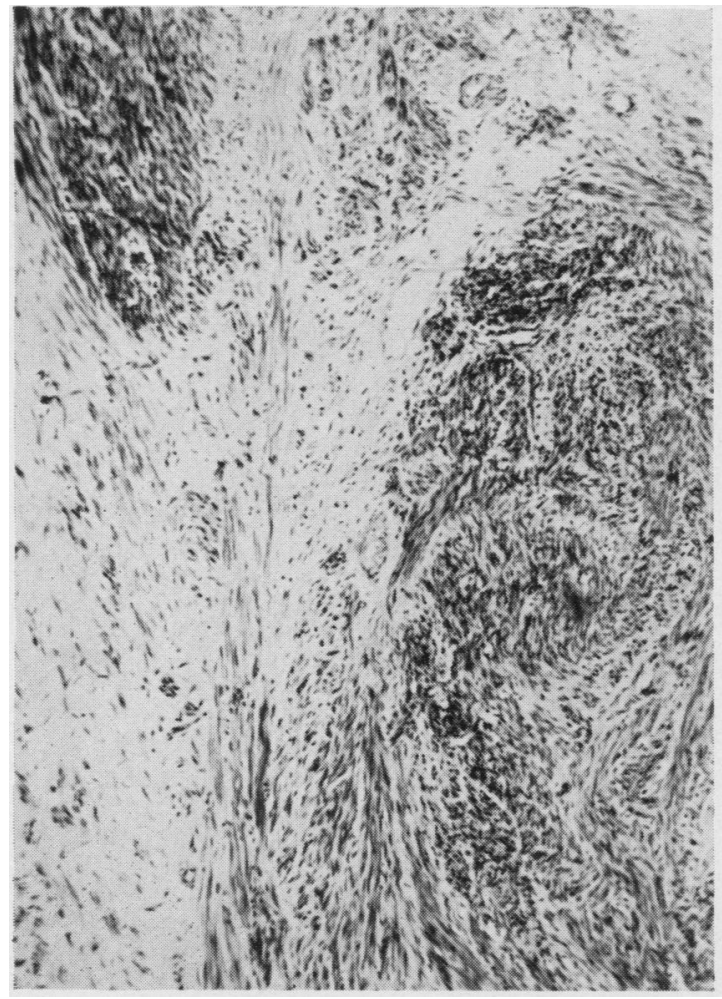

FIG. 3 (a).-Photomicrograph of Case 1. The irregular border of growth is visible, which displays the histological pattern of a smooth muscle tumour. Haematoxylin and eosin, $\times 78$.

well differentiated there are a few cells with large and somewhat anaplastic nuclei and there are mitoses on an average in one in every seven high-power fields (Fig. $3 a$ and $b$ ). The diagnosis is leiomyosarcoma.

The patient has been seen regularly since the tumour was removed and has remained perfectly well, without any evidence of recurrence. She was last examined on July 29, 1959, almost three years post-operatively. A full clinical review was satisfactory, and new chest films showed no evidence of a recurrence.

CASE 2.-An obese woman, aged 46, felt that she was getting somewhat short of breath and attended a mass miniature radiographic unit in January, 1953. The radiograph (Fig. $4 a$ and $b$ ) showed several rounded opacities in both lungs, with, in addition, a larger mass about $3 \mathrm{~cm}$. in diameter in the apex of the left lower lobe.

Apart from dyspnoea, which might well have been due to obesity, the only significant feature in her previous history was a total hysterectomy in 1937 for menorrhagia. There is no available report on the histology of the resected organ.

Lung biopsy was advised, and left thoracotomy was performed on May 9 (J.S.G.). All the tumours

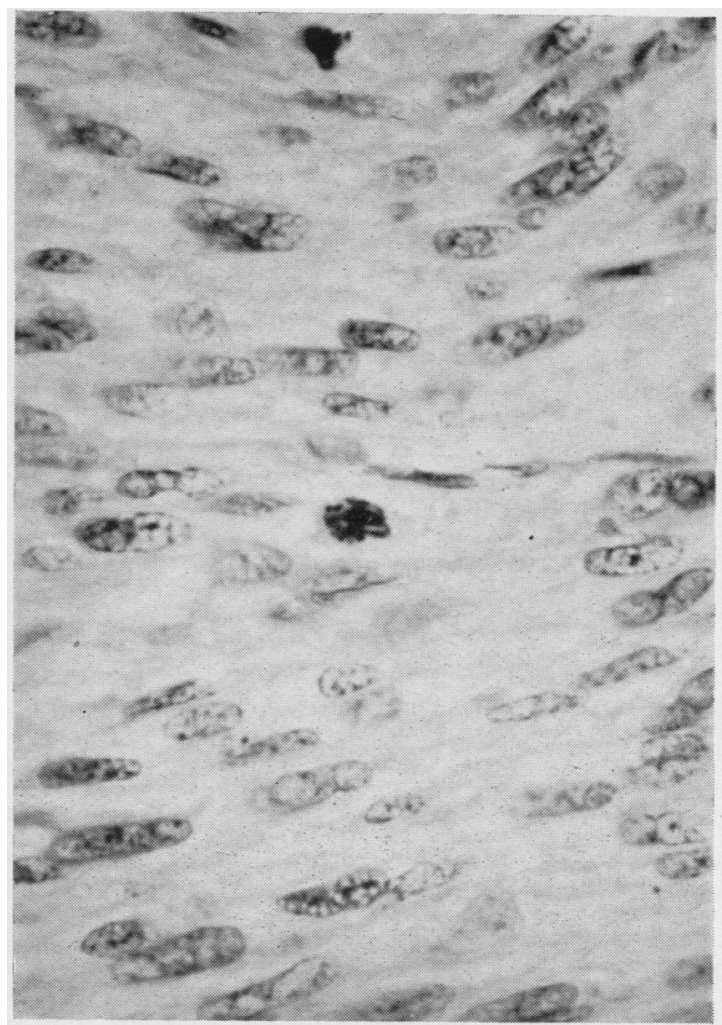

FIG. 3 (b).-Photomicrograph from Case 1 showing a mitosis. Haematoxylin and eosin, $\times 600$.

on the left side were found to be in the lung and to be mobile. Several of these were shelled out.

Pathological Report.-The specimen consisted of four rounded tumours measuring (1) $6.0 \times 5.0 \times 4.0$ cm., (2) $1.2 \times 1.0 \times 1.0 \mathrm{~cm}$., (3) and (4) $0.6 \mathrm{~cm}$. in diameter.

On transection the largest tumour was seen to be a firm, circumscribed, pale tumour.

Histological examination of the second largest tumour showed a typical smooth muscle tumour with an occasional mitosis.

The largest tumour is surrounded by rather oedematous stroma but no capsule. The tumour is a well-differentiated smooth muscle tumour, but the ill-defined border and the presence of some mitoses is suspicious of low-grade malignancy. There were no embryonal bronchial tubes in the sections of both specimens which might indicate an origin from multiple hamartomata (Fig. $5 a$ and $b$ ).

Dr. A. P. Stout reported on the largest tumour as follows:

With Masson's stain it is possible to see smooth muscle fibres in the cells, and the general configuration and arrangement of the cells are quite characteristic of a smooth muscle tumour. There are mitoses on 


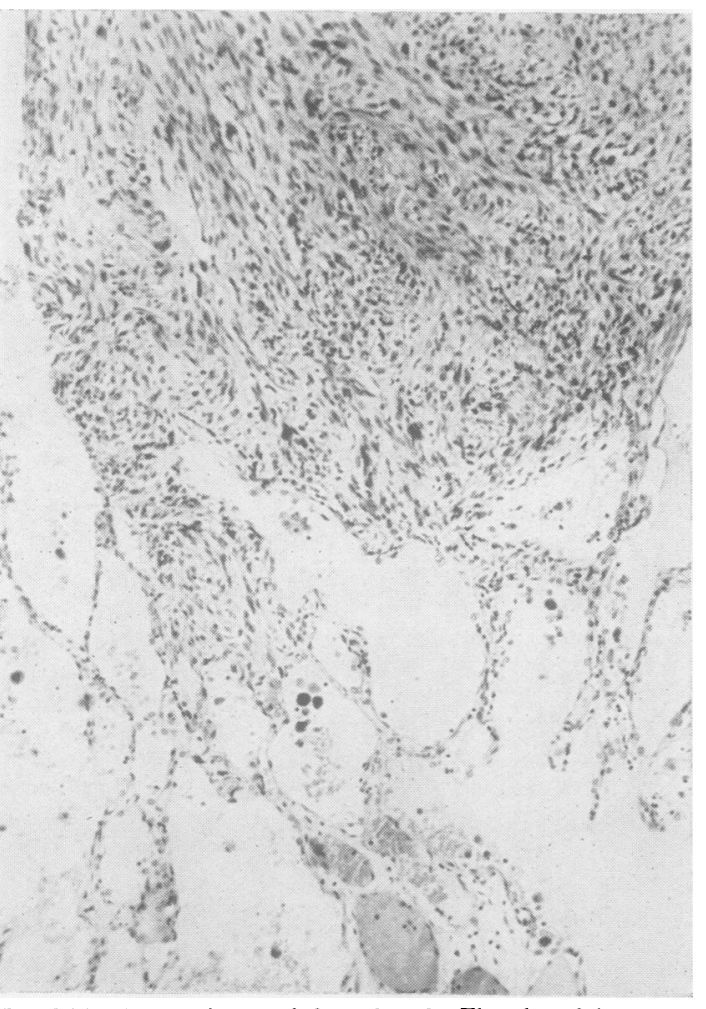

FIG. $5($ a).-Photomicrograph from Case 2. The edge of the tumour can be seen invading the lung parenchyma. Haematoxylin and eosin, $\times 78$.
FIG, 4 (b)

FIG. $4(a)$ and $(b)$-A $3 \mathrm{~cm}$. mass is visible in the apex of left lower lobe of Case 2. Two small ones can be seen in right lower zone.

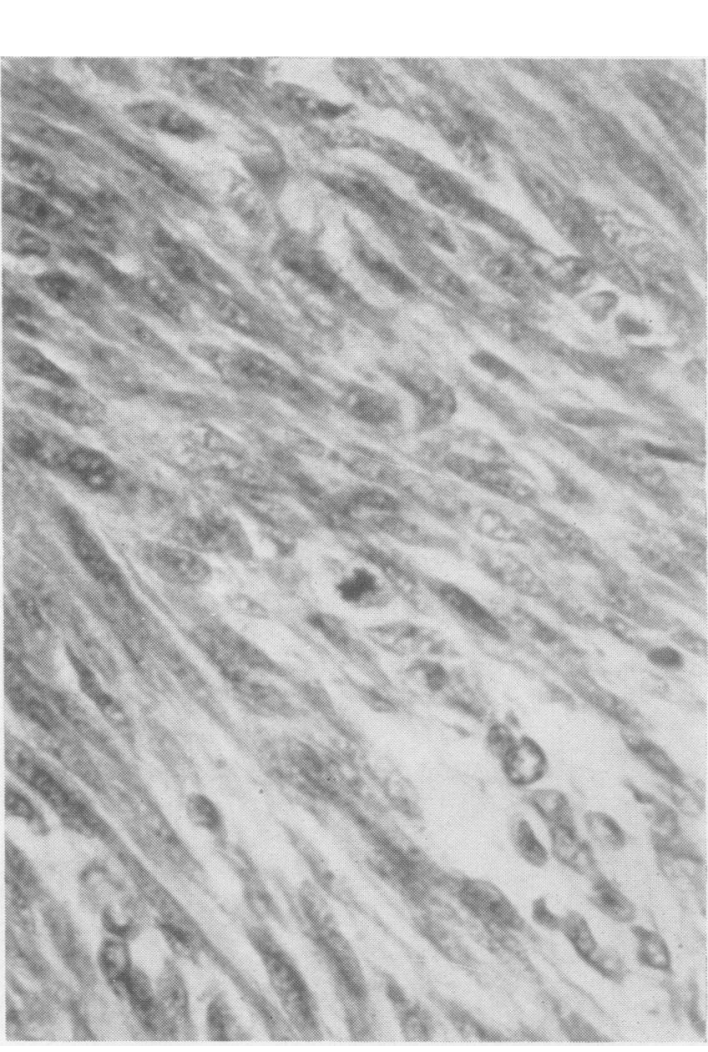

FIG. $5(b)$.- Photomicrograph from Case 2. A mitosis is seen amid typical smooth muscle cells. Haematoxylin and eosin, $\times 600$.

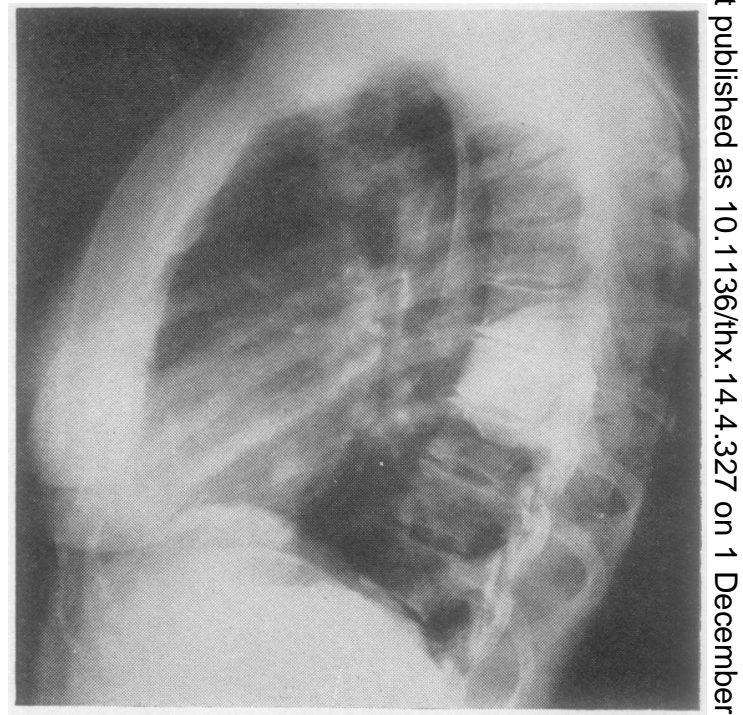

ज

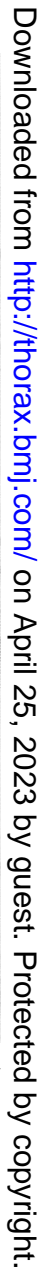


an average of one in every two high-power fields. In my opinion this tumour is a leiomyosarcoma.

This patient was last seen on March 2, 1959. She is very well, doing all her housework and is now much less dyspnoeic because she has deliberately lost 2 stones in weight. A new chest film on February 26, 1959, showed no alteration in the remaining tumours or recurrence at the sites of the excised ones.

\section{Discussion}

During recent years several surveys have been made of the literature on leiomyosarcoma of the lung, notably by Agnos and Starkey (1958). They found 14 cases of pulmonary leiomyoma and 18 cases of leiomyosarcoma had been described, and added two more cases of leiomyosarcoma themselves. Excellent summaries have also been given by Yacoubian, Connolly, and Wylie (1958) and Tocker, DeHaan, and Stofer (1957). A further case was added by Ochsner and Ochsner (1958). This patient was operated upon in April, 1936, probably the first pneumonectomy for leiomyosarcoma. Killingsworth, McReynolds, and Harrison (1953), reporting a similar tumour in a child aged 7 years, could only find nine cases previously reported in the chest in adults, and state that five of these were considered to be benign.

Stout has devoted much time and attention to the study of mesodermal tumours, and his papers on fibrosarcoma (1948) and leiomyosarcoma (Stout and Hill, 1958) describe beautifully in clear and concise terms the histological features of these groups of tumours.

The general use of carefully controlled differential stains has brought about a considerable improvement in the accuracy of diagnosis. In particular, the identification of typical cytoplasmic myofibrils in tumours enables a firm diagnosis of leiomyoma or well-differentiated leiomyosarcoma to be made with confidence.

There remains considerable confusion about malignancy in borderline cases. Exact criteria of malignancy are difficult to define and vary from one pathologist to another as will be realized by anyone who consults tumour panels.

Stout and Hill (1958) point out in their paper "Leiomyosarcoma of the Superficial Soft Parts" how difficulties have arisen in determining the degree of malignancy of leiomyosarcoma, both with regard to the unreliability of the criteria of numbers of bizarre cells or alternatively the numbers of mitoses which can be counted. On the whole myofibrils are frequent, with palisading of nuclei in $60 \%$ of cases, bizarre cells are to be found in a high proportion of leiomyosarcomas, with anaplasia in most, and mitotic figures in all cases, the figure varying from one in two to one in 10 high-power fields, an intermediate range of one in five being more frequent.

It was decided to refer the material reported in this paper to Dr. A. P. Stout, of Columbia University, for his authoritative opinion. He has informed us that his criteria for the diagnosis of leiomyosarcoma referred to above apply equally well to these tumours in the lung. In view of the small number of acceptable reports of pulmonary leiomyosarcomata in the literature, no similar confirmation is available elsewhere from the study of these lung tumours.

Some authorities have suggested that all females should be excluded from series of cases of leiomyosarcomata of the lungs, as the tumours may be secondary deposits from uterine neoplasms. This we regard as illogical. Neumann (1938) has pointed out that myomatous tumours have been described in any organ where muscle fibres are to be found outside the vascular system. It would appear reasonable to suppose that this is true of lung tissue, and furthermore one would expect to find muscle tumours arising most frequently in the region of the major bronchi where most muscle is present. This appears to be borne out by the cases so far described, primary tumours usually occurring near the lung hilum in contradistinction to secondary tumours which appear in the parenchyma. Each case, therefore, should be considered on its merits.

We consider that Case 1 is a primary pulmonary leiomyosarcoma, for, by good fortune and the kindness of Dr. C. S. Pike, of the Peace Memorial Hospital, Watford, we have received a full report and examined sections of the hysterectomy specimen from the 1951 operation, and there was no evidence of fibromyomata, or any form of malignancy in the uterus. The situation of the lung tumour was typical of a primary arising in the lower lobe main bronchus, and was visible on bronchoscopy. No further tumours have developed since the lobectomy was carried out three years previously.

This case conforms to the description of Yacoubian et al. (1958) in that the local lymph glands were not involved, but that the tumour was extending rather along the course of the pulmonary vein to encroach on the pericardium, thus demonstrating the tendency of these tumours to spread by intravascular extension.

Case 2 is remarkable and presents atypical features that are strikingly different from Case 1. Whereas in Case 1 the tumour was solitary and central, in Case 2 multiple tumours were scattered 
through both lungs. These were discovered 16 years after a hysterectomy, and the residual ones left undisturbed at the thoracotomy in 1953 have remained unchanged radiologically for a further six years. It is now 22 years since this patient's hysterectomy and this would seem to exclude the possibility of there being metastatic tumours from a hypothetical uterine malignancy. Furthermore, since they appear to have remained quiescent over a period of six years after the thoracotomy, it appears unlikely that any of these residual tumours were sarcomatous. Histological preparations of the largest tumour removed from Case 2 reveal a picture indistinguishable from leiomyosarcoma, whereas the smaller tumours were apparently benign leiomyomas. It appears, therefore, that this case is one of multiple leiomyoma in which malignant change has developed in one of the tumours to give a typical leiomyosarcoma, and that this tumour has been completely removed simply by " shelling out "instead of by the classical procedure of lobectomy or pneumonectomy.

The patient will be closely observed to detect any change in the radiographs which might indicate malignant change in any of the tumours which remain. As these multiple tumours are bilateral, lobectomy or pneumonectomy can only be considered when and if changes are observed.

\section{SUMMARY}

Two cases of leiomyosarcoma of the lungs in women are reported. In Case 1 a classical leiomyosarcoma arising near a large bronchus $\stackrel{\overparen{D}}{\AA}$ was removed. In Case 2 a leiomyosarcoma was shelled out with other leiomyomata from a lung with multiple bilateral pulmonary shadows.

Both cases are regarded as primary lung $\overrightarrow{\vec{\omega}}$ tumours, and the management of Case 2 is $\mathscr{O}$ discussed.

We should like to record our appreciation for information on Case 1 kindly supplied by Mr. S. A. $\dot{\omega}$ Scorer, M.R.C.O.G., and Dr. C. S. Pike, of the Peace N Memorial Hospital, Watford, and to acknowledge our indebtedness to Mr. J. H. Kugler, A.I.M.L.T., for the photomicrographs. Finally we are most grateful to Dr. A. P. Stout, of Columbia University, New York, for advice and encouragement in this study.

REFERENCES

Agnos, J. W. and Starkey, G. W. B. (1958). New Engl. J. Med., 258, 12.

Killingsworth, W. P., McReynolds, G. S., and Harrison, A. W. (1953). J. Pediat., 42, 466.

Neumann, R. (1938). Frankfurt. Z. Path., 52, 576.

Ochsner, A., and Ochsner, S. (1958). J. thorac. Surg., 35, 768.

Stout, A. P. (1948). Cancer, 1, 30.

Tocker, A. M., DeHaan, C., and Stofer, B. E. (1957). Dis. Chest, 31, 328.

Yacoubian, H., Connolly, J. E., and Wylie, R. H. (1958). Ann. ڤ్ Surg., 147, 116. 